\title{
ON STRONGLY ASSOCIATIVE (SEMI)HYPERGROUPS
}

\author{
F. JANTANi ${ }^{1}$, M. JAFARPour ${ }^{2}$, V. LEOREANU-FoteA ${ }^{3}$ \\ ${ }^{1}$ Mathematics Department, Vali-e-Asr University of Rafsanjan, \\ Rafsanjan, Iran, \\ jantani_math@yahoo.com \\ ${ }^{2}$ Mathematics Department, Vali-e-Asr University of Rafsanjan, \\ Rafsanjan, Iran, \\ m.j@mail.vru.ac.ir \\ ${ }^{3}$ Faculty of Mathematics, Al.I. Cuza University, \\ 6600 Iasi, Romania, \\ foteavioleta@gmail.com
}

\begin{abstract}
In this paper, we introduce the notion of a strongly associative hyperoperation that we call SASS and obtain a new class of (semi)hypergroups. Moreover, we study this concept in the context of $K_{H}$-semihypergroups, complete hypergroups, polygroups and Rosenberg hypergroups.

Key words and Phrases: Strongly associative hyperoperation, polygroups, complementable semihypergroup, Rosenberg hypergroup.
\end{abstract}

\begin{abstract}
Abstrak. Pada makalah ini kami memperkenalkan ide suatu hiperoperasi asosiatif kuat yang kami nyatakan dengan SASS dan mendapatkan suatu kelas baru (semi)hipergrup. Lebih jauh, kami mempelajari konsep di atas dalam konteks $K_{H^{-}}$ semihipergrup, hipergrup lengkap, poligrup dan hipergrup Rosenberg.

Kata kunci: Hiperoperasi asosiatif kuat, poligrup, semihipergrup dapat dikomplemen, hipergrup Rosenberg.
\end{abstract}

\section{INTRODUCTION}

Hyperstructures state a natural extension of classical algebraic structures and they were introduced in 1934 by the French mathematician Marty [11]. A set $H$ endowed with a mapping $\circ: H \times H \longrightarrow P^{*}(H)$, named hyperoperation, is called hypergroupoid, where $P^{*}(H)$ denotes the set of all non-empty subsets of $H$. The image of a pair $(x, y)$ is denoted by $x \circ y$ or $x y$. If $x \in H$ and $A, B$ are non-empty subsets of $H$, then by $A \circ B, A \circ x$ and $x \circ B$ we mean $A \circ B=\cup\{a b \mid a \in A, b \in$

2000 Mathematics Subject Classification: 20N20.

Received: 03-01-2017, revised: 20-02-2017, accepted: 21-02-2017. 
$B\}, A \circ x=A \circ\{x\}$ and $x \circ B=\{x\} \circ B$, respectively. A semihypergroup is a hypergroupoid $(H, \circ)$ such that for all $x, y$ and $z$ in $H$ we have $(x \circ y) \circ z=x \circ(y \circ z)$ and a hypergroup is a semihypergroup which satisfies the reproductive axiom. i.e., for all $x \in H, H \circ x=x \circ H=H$. Resent decades numerous papers and books on algebraic hyperstructures have been published that surveys of the researches can be found in the books of Corsini [4], Corsini and Leoreanu [3], Vougiouklis [13], Davvaz and Leoreanu [6] and Davvaz [8, 7]. We know that the quotient of a group with respect to an invariant subgroup is a group. Marty states that the quotient of a group with respect to any subgroup is a hypergroup. Generally the quotient of a group with respect to any partition (or equivalently to any equivalence relation) is an $H_{v}$-group. This is the motivation to introduce the $H_{v^{-}}$structures [14]. $H_{v^{-}}$ structures for the first time introduced by Vougiouklis at the Fourth AHA congress (1990) [14]. The concept of $H_{v}$-structures constitutes a generalization of the wellknown algebraic hyperstructures (hypergroup, hyperring, hypermodule and so on). Actually some axioms concerning $H_{v}$-structures such as the associative law, the distributive law and so on are replaced by their corresponding weak axioms. Some basic definitions and theorems about $H_{v}$-structures can be found in [13]. Let $(H, \circ)$ be a hypergroupoid. The hyperoperation "o "on $H$ is called weak associative (WASS) if for all $x, y, z \in H,(x \circ y) \circ z \cap x \circ(y \circ z) \neq \emptyset$. The hyperstructure $(H, \circ)$ is called an $H_{v}$-semigroup if "० "is weak associative. An $H_{v}$-semigroup is called an $H_{v}$-group if for all $a \in H, a \circ H=H \circ a=H$. For more definitions and applications on $H_{v}$-structures, see the books $[3,4,6]$ and papers as $[2,9,15,16,17]$. A semihypergroup $H$ is regular if it has at least one identity and each element has at least one inverse. A regular hypergroup $(H, \circ)$ is called reversible if it satisfies the following conditions, for all $(x, y, a) \in H^{3}$ :

(1) If $y \in a \circ x$, an inverse $a^{\prime}$ of $a$ exists such that $x \in a^{\prime} \circ y$,

(2) If $y \in x \circ a$, an inverse $a^{\prime \prime}$ of $a$ exists such that $x \in y \circ a^{\prime \prime}$.

If $H$ is regular and $a \in H$, we denote by $E$ the set of bilateral identities and by $i(a)$ the set of inverses of $a$. A semihypergroup $H$ is complete if it satisfies one of the following equivalent conditions:

(1) $\forall(x, y) \in H^{2}, \forall a \in x \circ y, \mathcal{C}(a)=x \circ y$, where $\mathcal{C}(a)$ denotes the complete closure of $a$

(2) $\forall(x, y) \in H^{2}, \mathcal{C}(x \circ y)=x \circ y$,

(3) $\forall(n, m) \in \mathbf{N}^{2}, m, n \geq 2, \forall\left(x_{1}, \ldots, x_{n}\right) \in H^{n}, \forall\left(y_{1}, \ldots, y_{m}\right) \in H^{m}$, the following implication is valid:

$$
\Pi_{i=1}^{n} x_{i} \cap \Pi_{j=1}^{m} y_{j} \neq \emptyset \Rightarrow \Pi_{i=1}^{n} x_{i}=\Pi_{j=1}^{m} y_{j} .
$$

In this paper, we introduce the notion of a strongly associative hyperoperation called SASS hyperoperation and obtain a new class of (semi)hypergroups which we call strongly associative (semi)hypergroups. Our first aim is to investigate this concept for some semihypergroups such $K_{H}$-semihypergroups, complete hypergroups, polygroups, complement hypergroups and Rosenberg hypergroups. Moreover, we introduce a class of non-strongly associative hypergroups which are derived from groups. 
Theorem 1.1. ( $[3], 138)$ A semihypergroup $(H, \circ)$ is complete if it can be written as a union $H=\cup_{s \in S} A_{s}$ of its subsets, where $S$ and $A_{s}$ satisfy the conditions:

(1) $(S, \cdot)$ is a semigroup;

(2) for all $(s, t) \in S^{2}$, where $s \neq t$, we have $A_{s} \cap A_{t}=\emptyset$;

(3) if $(a, b) \in A_{s} \times A_{t}$, then $a \circ b=A_{s t}$.

Theorem 1.2. ( [3], 141) If $H$ is a complete hypergroup, then $H$ is regular and reversible.

\section{On Strongly Associative Hyperoperations}

In this section, we present some definitions and examples on strongly associative hyperstructures and we introduce strongly associative semihypergroups.

Definition 2.1. Let $H$ be a non-empty set and $\circ: H \times H \longrightarrow \mathcal{P}^{*}(H)$ be a hyperoperation. Then

(1) $\circ$ is called left strongly associative if for all $x, y, z \in H$ and for all $t \in y \circ z$, there exists $s \in x \circ y$ such that $x \circ t=s \circ z$,

(2) $\circ$ is called right strongly associative if for all $x, y, z \in H$ and for all $t \in x \circ y$, there exists $s \in y \circ z$ such that $t \circ z=x \circ s$,

(3) $\circ$ is strongly associative or for simplicity SASS if it is left and right strongly associative.

Definition 2.2. A hypergroupoid $(H, \circ)$ is called left (right) strongly associative if the hyperoperation $\circ$ is left (right) strongly associative. $(H, \circ)$ is called strongly associative if $\circ$ is strongly associative.

Remark 2.1. The group operation is strongly associative.

Example 2.3. Let $H$ be a hypergroup, defined as follows:

\begin{tabular}{c|ccc}
$\cdot$ & $e$ & $a$ & $b$ \\
\hline$e$ & $\{e, a\}$ & $\{e, b\}$ & $\{e, b\}$ \\
$a$ & $\{e, b\}$ & $\{e, a\}$ & $\{e, a\}$ \\
$b$ & $\{e, b\}$ & $\{e, a\}$ & $\{e, a\}$
\end{tabular}

The hyperoperation "."is strongly associative and $H$ is a strongly associative hypergroup.

Example 2.4. Let $H$ be a hypergroup defined by the following table:

\begin{tabular}{c|ccc}
$\cdot$ & $x$ & $y$ & $z$ \\
\hline$x$ & $x$ & $\{x, y, z\}$ & $\{x, y, z\}$ \\
$y$ & $\{x, y, z\}$ & $\{y, z\}$ & $\{y, z\}$ \\
$z$ & $\{x, y, z\}$ & $\{y, z\}$ & $\{y, z\}$
\end{tabular}

Then the hyperoperation " "is not a left strongly associative nor a right strongly associative and so $H$ is not a strongly associative hypergroup. 
Example 2.5. Consider $H=\{a, b, c\}$ and define. on $H$ with the help of the following table:

\begin{tabular}{c|ccc}
$\cdot$ & $a$ & $b$ & $c$ \\
\hline$a$ & $a$ & $\{a, b\}$ & $H$ \\
$b$ & $a$ & $\{a, b\}$ & $H$ \\
$c$ & $H$ & $H$ & $H$
\end{tabular}

$H$ is a right strongly associative hypergroup and it is not a left strongly associative hypergroup.

Proposition 2.6. If the hyperoperation o is strongly associative on $H$, then $(H, \circ)$ is a semihypergroup.

Proof. Let $\circ$ be a SASS hyperoperation on $H$ and $(x, y, z) \in H^{3}$. Then we have $\{x \circ t \mid t \in y \circ z\}=\{s \circ z \mid s \in x \circ y\}$. Hence $(x \circ y) \circ z=x \circ(y \circ z)$.

Proposition 2.7. If $H$ is a strongly associative hypergroup and there exists $x \in H$ such that for all $y \in H, y \circ x=H$, then $H$ is a total hypergroup.

Proof. Suppose that $\alpha, y \in H$. According to the strongly associativity of $H$, for all $t \in y \circ x, \alpha \circ t=H$, since for all $s \in \alpha \circ y$, we have $s \circ x=H$. Therefore $H$ is a total hypergroup.

Similarly, if $H$ is a strongly associative hypergroup and there exists $x \in H$ such that for all $y \in H, x \circ y=H$, then $H$ is a total hypergroup.

Example 2.8. Let $H=\{e, a, b\}$. We define the following hypergroup structure on $H$ :

\begin{tabular}{c|ccc}
$\circ$ & $e$ & $a$ & $b$ \\
\hline$e$ & $\{e, a\}$ & $H$ & $H$ \\
$a$ & $H$ & $\{e, a\}$ & $\{e, a\}$ \\
$b$ & $H$ & $\{e, a\}$ & $\{e, a\}$
\end{tabular}

Then $H$ is a strongly associative hypergroup which is not a total hypergroup.

Proposition 2.9. Let $(H, \circ)$ be a strongly associative hypergroup and $x, y, z \in H$. If $y \circ z=\{t\}$ and $\{u, v\} \subseteq x \circ y$, then $u \circ z=v \circ z$.

Proof. Since $H$ is e strongly associative, $x \circ t=s \circ z$ for all $s \in x \circ y$. Therefore $u \circ z=v \circ z$.

Corollary 2.10. If $(H, \circ)$ is a hypergroup and there exist $x, y, z \in H$ such that $y \circ z=\{t\}$ and $x \circ y=H$, then $H$ is not a strongly associative hypergroup.

Proof. Let $(H, \circ)$ be a hypergroup. By the previous proposition, for all $\alpha \in H$, $\alpha \circ z=H$ and by proposition 2.7. $H$ is a total hypergroup, a contradiction.

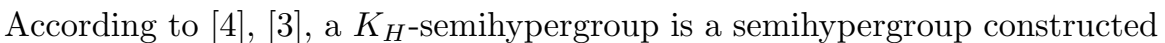
from a semihypergroup $(H, \circ)$ and a family $\{A(x)\}_{x \in H}$ of non-empty and mutually 
disjoint subsets of $H$. Set $K_{H}=\cup_{x \in H} A(x)$ and define the hyperoperation $*$ on $K_{H}$ as follows:

$$
\forall(a, b) \in K_{H}^{2} ; a \in A(x), b \in A(y), a * b=\cup_{z \in x \circ y} A(z) .
$$

Theorem 2.11. [3] $(H, \circ)$ is a hypergroup if and only if $\left(K_{H}, *\right)$ is a hypergroup.

Theorem 2.12. If $H$ is a semihypergroup, then $K=K_{H}$ is a strongly associative semihypergroup if and only if $H$ is a strongly associative semihypergroup.

Proof. Let $H$ be a strongly associative semihypergroup and $\{a, b, c\} \subseteq K$. Then there exist $\{x, y, z\} \subseteq H$ such that $a \in A(x), b \in A(y), c \in A(z)$. Let $t \in b * c$. There exists $\alpha \in y \circ z$ such that $t \in A_{\alpha}$. Since $H$ is strongly associative, there exists $r \in x \circ y$ such that $x \circ \alpha=r \circ z$. Let $s \in A_{r}$. We have $a * t=\cup_{l \in x \circ \alpha} A_{l}=\cup_{l \in r \circ} z A_{l}=s * c$ and so $K=K_{H}$ is a strongly associative semihypergroup.

For the converse, let $K=K_{H}$ be a strongly associative semihypergroup and $\{x, y, z\} \subseteq H$. Let $\{a, b, c\} \subseteq K$ be such that $a \in A(x), b \in A(y), c \in A(z)$ and $t \in y \circ z$. Thus $A_{t} \subseteq \cup_{l \in y \circ z} A_{l}=b * c$. Let $t^{\prime} \in A_{t} \subseteq b * c$. There exists $s^{\prime} \in a * b$ such that $a * t^{\prime}=s^{\prime} * c$ and thus $\cup_{\beta \in x \circ t} A_{\beta}=\cup_{\tau \in s \circ z} A_{\tau}$, where $s \in H$ and $s^{\prime} \in A_{s}$. So $x \circ t=s \circ z$, since the family $\{A(x)\}_{x \in H}$ contains mutually disjoint subsets of $H$.

Corollary 2.13. If $(H, \circ)$ is a complete semihypergroup, then it is strongly associative.

Notice that there are semihypergroups that are not complete, but they are strongly associative. Example 2.3. presents such a semihypergroup.

Let $\left(H_{1}, \cdot\right)$ and $\left(H_{2}, \circ\right)$ be two hypergroups. On $H_{1} \times H_{2}$ we can define a hyperproduct as follows: $\left(x_{1}, y_{1}\right) *\left(x_{2}, y_{2}\right)=\left\{(x, y) \mid x \in x_{1} \cdot x_{2}, y \in y_{1} \circ y_{2}\right\}$. This is the direct product of $H_{1}$ and $H_{2}$ and it is clearly a hypergroup.

Theorem 2.14. If $H_{1}$ and $H_{2}$ are two strongly associative hypergroups, then $H_{1} \times$ $\mathrm{H}_{2}$ is a strongly associative hypergroup.

Proof. Let $\left\{\left(x_{1}, x_{2}\right),\left(y_{1}, y_{2}\right),\left(z_{1}, z_{2}\right)\right\} \subseteq H_{1} \times H_{2}$ and $\left(t_{1}, t_{2}\right) \in\left(y_{1}, y_{2}\right) *\left(z_{1}, z_{2}\right)$. Therefore $t_{1} \in y_{1} \cdot z_{1}$ and $t_{2} \in y_{2} \circ z_{2}$. Since $H_{1}$ and $H_{2}$ are strongly associative, there exist $s_{1} \in x_{1} \cdot y_{1}$ and $s_{2} \in x_{2} \circ y_{2}$ such that $x_{1} \cdot t_{1}=s_{1} \cdot z_{1}$ and $x_{2} \circ t_{2}=s_{2} \circ z_{2}$. Thus $\left(t_{1}, t_{2}\right) \in\left(y_{1}, y_{2}\right) *\left(z_{1}, z_{2}\right)$ and $\left(x_{1}, x_{2}\right) *\left(t_{1}, t_{2}\right)=\left(s_{1}, s_{2}\right) *\left(z_{1}, z_{2}\right)$. Therefore $H_{1} \times H_{2}$ is a left strongly associative hypergroup.

Similarly, it can be checked that $H_{1} \times H_{2}$ is a right strongly associative hypergroup and this completes the proof.

\section{On Strongly Associative Complementable (Semi)Hypergroups}

In this section, we show that the complement hypergroup of a complete hypergroup is strongly associative and a polygroup is strongly associative if and only if it is group. 
Let $(H, \circ)$ be a semihypergroup such that $x \circ y \neq H$, for all $(x, y) \in H^{2}$.

According to [1], the complement of $(H, \circ)$ is the hypergroupoid $\left(H, \circ^{c}\right)$ endowed with the complement hyperoperation: $x \circ^{c} y=H-x \circ y$.

The semihypergroup $(H, \circ)$ is complementable if its complement $\left(H, \circ^{c}\right)$ is a semihypergroup too.

$\left(H, \circ^{c}\right)$ is called the complement semihypergroup of $(H, \circ)$.

Theorem 3.1. ([1], 5.2.) The hypergroup $K_{H}$ is complementable if and only if $H$ is complementable.

Proposition 3.2. All groups of order at least 2 are complementable.

Proof. Let $(G, \cdot)$ be a group. If $|G|=2$ then $\left(G, \cdot{ }^{c}\right)$ is a group, too. Now suppose that $|G| \geq 3$ and $x, y, z \in G$. We have

$$
\left(x \cdot{ }^{c} y\right) \cdot{ }^{c} z=\bigcup_{u \in x \cdot{ }^{c} y} u \cdot{ }^{c} z \supseteq u_{1} \cdot{ }^{c} z \cup u_{2} \cdot{ }^{c} z
$$

where $u_{1} \neq u_{2}$ and $u_{1}, u_{2} \in x \cdot{ }^{c} y$. Since $(G, \cdot)$ is group, $u_{1} \cdot{ }^{c} z \cup u_{2} \cdot{ }^{c} z=G$. Hence $\left(x \cdot{ }^{c} y\right) \cdot{ }^{c} z=G$ and similarly $x \cdot{ }^{c}\left(y \cdot{ }^{c} z\right)=G$, whence $\left(x \cdot{ }^{c} y\right) \cdot{ }^{c} z=x \cdot{ }^{c}\left(y \cdot{ }^{c} z\right)$.

Corollary 3.3. All non-total complete hypergroups of order at least 2 are complementable.

Proof. It follows by the previous proposition and Theorems 1.1 and 3.1.

Theorem 3.4. The complement of a complete hypergroup is a strongly associative hypergroup.

Proof. Let $(H, \circ)$ be a non-total complete hypergroup. If $|H|=2$ then $\left(H, \circ^{c}\right)$ is a group and so, it is strongly associative.

Now suppose that $\{x, y, z\} \subseteq H$ and $t \in y \circ^{c} z$, so $t \notin y \circ z$. If $s \in x \circ t \circ z^{\prime}$, where $z^{\prime} \in i(z)$ (the set of inverse elements of $z$ in $(H, \circ)$ ) it follows that $s \in x \circ^{c} y$. If $s \notin x \circ^{c} y$, then $s \in x \circ y \cap x \circ t \circ z^{\prime}$. Since $H$ is complete, $x \circ y=x \circ t \circ z^{\prime}$ and so

$$
\begin{aligned}
x^{\prime} \circ x \circ y & =x^{\prime} \circ x \circ t \circ z^{\prime} \\
& =e \circ t \circ z^{\prime} \\
& =t \circ z^{\prime}
\end{aligned}
$$

where $x^{\prime} \in i(x)$, therefore $t \in y \circ z$ which is a contradiction.

Therefore, $x \circ^{c} t=s \circ^{c} z$, therefore $\left(H, \circ^{c}\right)$ is strongly associative on the right. Similarly it is strongly associative on the left and so it is a strongly associative hypergroup.

There exist strongly associative semihypergroups of which complement semihypergroups are not strongly associative. The next example is such a semihypergroup. 
Example 3.5. [1] Set $H=\{e, a, b\}$. Consider the semihypergroup $(H, \circ)$ endowed with the hyperoperation $\circ$ defined as follows:

\begin{tabular}{c|ccc}
$\circ$ & $e$ & $a$ & $b$ \\
\hline$e$ & $\{a, b\}$ & $b$ & $b$ \\
$a$ & $b$ & $b$ & $b$ \\
$b$ & $b$ & $b$ & $b$
\end{tabular}

Notice that $H$ is a strongly associative complementable semihypergroup, of which complement, defined as follows

\begin{tabular}{c|ccc}
$\circ^{c}$ & $e$ & $a$ & $b$ \\
\hline$e$ & $e$ & $\{e, a\}$ & $\{e, a\}$ \\
$a$ & $\{e, a\}$ & $\{e, a\}$ & $\{e, a\}$ \\
$b$ & $\{e, a\}$ & $\{e, a\}$ & $\{e, a\}$
\end{tabular}

is a semihypergroup, which it is not strongly associative.

Corollary 3.6. If $(G, \cdot)$ is a group, then $\left(G, \cdot^{c}\right)$ is a strongly associative hypergroup.

A polygroup is a system $\zeta=<P, \cdot,^{-1}>$, where $e \in P$ and ${ }^{-1}$ is a unitary operation on $P$, while $\cdot$ maps $P \times P$ into the set of all non-empty subsets of $P$, and the following axioms hold for all $x, y, z \in P$ :

(P1) $(x \cdot y) \cdot z=x \cdot(y \cdot z)$;

(P2) $e \cdot x=x \cdot e=x$;

(P3) $x \in y \cdot z$ implies $y \in x \cdot z^{-1}$ and $z \in y^{-1} \cdot x$ (see [7]).

[7] contains some definitions and results about polygroups.

Lemma 3.7. A polygroup $\left\langle P, \cdot, e,,^{-1}\right\rangle$ is a group if and only if for all element a of $P,\left|a \cdot a^{-1}\right|=1$.

Proof. Set $\{c, b, \alpha, \beta\} \subseteq P$ and $\{\alpha, \beta\} \subseteq c . b$. We have

$$
\begin{aligned}
& \alpha \in c . b \\
\Rightarrow & b \in c^{-1} \alpha \\
\Rightarrow & \beta \in c . b \subseteq c c^{-1} \alpha=\alpha \\
\Rightarrow & \alpha=\beta .
\end{aligned}
$$

Thus, for all $(c, b) \in P^{2},|c \cdot b|=1$ and hence $P$ is a group.

Proposition 3.8. A polygroup $P$ is strongly associative if and only if it is a group.

Proof. Suppose that $\left\langle P, \cdot, e,^{-1}\right\rangle$ is a strongly associative polygroup, which it is not a group. By the above lemma, there exist $f, a \in P$ such that $f \cdot f^{-1}=\{e, a\}$. We have $a \in f \cdot f^{-1}, e \cdot f=f$ and $e \cdot a \neq f \cdot f^{-1}$. In other words, $P$ is not a strongly associative polygroup, which is false. The converse is obvious. 


\section{On Strongly Rosenberg Hypergroups}

In this section, we prove that a Rosenberg hypergroup is strongly associative if and only if it is a total hypergroup. In [12], Rosenberg associated a partial hypergroupoid $H_{\rho}=\left(H, \circ_{\rho}\right)$ with a binary relation $\rho$ defined on a set $H$, in the following way:

$$
x \circ_{\rho} x=\{z \in H \mid(x, z) \in \rho\}, x \circ_{\rho} y=x \circ_{\rho} x \cup y \circ_{\rho} y,
$$

for all $(x, y) \in H^{2}$.

Theorem 4.1. [12] $H_{\rho}$ is a hypergroup if and only if

(1) $\rho$ has full domain;

(2) $\rho$ has full range;

(3) $\rho \subset \rho^{2}$;

(4) If $(a, x) \in \rho^{2}$, then $(a, x) \in \rho$, whenever $x$ is an outer element of $\rho$.

An element $x \in H$ is called outer element of $\rho$ if there exists $h \in H$ such that $(h, x) \notin \rho^{2}$.

Lemma 4.2. Let $H$ be a non-empty set such that $|H| \geq 2$ and

$$
\rho=\{(x, x) \mid x \in H\} .
$$

Then the associated Rosenberg hypergroup $\left(H, \circ_{\rho}\right)$ of $\rho$ is not a strongly associative hypergroup.

Proof. Let $x, y, z \in H$. We have $x \circ y \neq x \circ z$ and $x \circ y \neq y \circ z$, whence it follows the conlusion.

Theorem 4.3. A Rosenberg hypergroup $\left(H, \circ_{\rho}\right)$ is strongly associative if and only if it is a total hypergroup.

Proof. Let $\left(H, \circ_{\rho}\right)$ be a strongly associative Rosenberg hypergroup. $H$ has full range, so for all element $y \in H$ there exists $x \in H$ such that $y \in x \circ x$. Therefore $y \in x \circ y$. Since $H$ is strongly associative, there exists $\beta \in x \circ y$ such that $y \circ y=$ $x \circ \beta=x \circ x \cup \beta \circ \beta$. Therefore $x \circ x \subseteq y \circ y$ and so $y \in y \circ y$. Hence we obtain that $\rho$ is reflexive. By Lemma 4.2. there are $x, y \in H(x \neq y)$ such that $y \in x \circ x$. Hence $x \circ x \subseteq y \circ y$ and $\{x, y\} \subseteq x \circ x \cap y \circ y$.

Now set $z \in H$. We have $x \in y \circ z$. Since $H$ is strongly associative, there exists $\alpha \in x \circ y$ such that $x \circ x=\alpha \circ z$. Therefore $z \in x \circ x$ and so $H=x \circ x$ and $H=y \circ y$. Set $\alpha \in H-\{x, y\}$. Then $\alpha \in x \circ x$. There exists $a \in x \circ \alpha$ such that $\alpha \circ \alpha=x \circ a$, consequently $x \circ x \subseteq \alpha \circ \alpha$ and so $\alpha \circ \alpha=H$. Thus we conclude that $H$ is a total hypergroup. The converse is obvious.

\section{Derived Non-Strongly Associative Hypergroups from Groups}

Definition 5.1. [5] We say that two partial hypergroupoids $\left(H, \circ_{1}\right)$ and $\left(H, \circ_{2}\right)$ are weak mutually associative or w.m.a., if for all $(x, y, z) \in H^{3}$, we have:

$$
\left(x \circ_{1} y\right) \circ_{2} z \cup\left(x \circ_{2} y\right) \circ_{1} z=x \circ_{1}\left(y \circ_{2} z\right) \cup x \circ_{2}\left(y \circ_{1} z\right) \text {. }
$$


Definition 5.2. We say that two partial hypergroupoids $\left(H, \circ_{1}\right)$ and $\left(H, \circ_{2}\right)$ are mutually associative or m.a., if for all $(x, y, z) \in H^{3}$, we have $\left(x \circ_{1} y\right) \circ_{2} z=$ $x \circ_{2}\left(y \circ_{1} z\right)$ and $\left(x \circ_{2} y\right) \circ_{1} z=x \circ_{1}\left(y \circ_{2} z\right)$. follows:

If $(G, \cdot)$ and $(G, \circ)$ are groupoids, we can define a hyperoperation $\star$ on $G$ as

$$
x \star y=\{x \cdot y, x \circ y\},
$$

for all $(x, y) \in G^{2}$.

From now on, we call $\star$ the derived hyperoperation from $(G, \cdot)$ and $(G, \circ)$.

Proposition 5.3. If $(G, \cdot)$ and $(G, \circ)$ are groups, such that their operations are mutually associative, then the derived hyperoperation from $(G, \cdot)$ and $(G, \circ)$ is strongly associative.

Proof. Set $(x, y, z) \in G^{3}$. Then $(x \star y) \star z=\cup\{(x \cdot y) \star z,(x \circ y) \star z\}$ and $x \star(y \star z)=$ $\cup\{x \star(y \cdot z), x \star(y \circ z)\}$. Since the groups $(G, \cdot)$ and $(G, \circ)$ are m.a we conclude that $(x \cdot y) \star z=x \star(y \cdot z)$ and $(x \circ y) \star z=x \star(y \circ z)$, hence $\star$ is a strongly associative hyperoperation.

Example 5.4. Set $G=\{e, a, b, c\}$ endowed with the following operations:

\begin{tabular}{c|cccc}
$\cdot$ & $e$ & $a$ & $b$ & $c$ \\
\hline$e$ & $e$ & $a$ & $b$ & $c$ \\
$a$ & $a$ & $b$ & $c$ & $e$ \\
$b$ & $b$ & $c$ & $e$ & $a$ \\
$c$ & $c$ & $e$ & $a$ & $b$
\end{tabular}

\begin{tabular}{c|cccc}
$\circ$ & $e$ & $a$ & $b$ & $c$ \\
\hline$e$ & $a$ & $b$ & $c$ & $e$ \\
$a$ & $b$ & $c$ & $e$ & $a$ \\
$b$ & $c$ & $e$ & $a$ & $b$ \\
$c$ & $e$ & $a$ & $b$ & $c$
\end{tabular}

$(G, \cdot)$ and $(G, \circ)$ are groups and their operations are mutually associative. Moreover, the derived hyperoperation from $(G, \cdot)$ and $(G, \circ)$, described bellow, is strongly associative.

\begin{tabular}{c|cccc}
$\star$ & $e$ & $a$ & $b$ & $c$ \\
\hline$e$ & $e, a$ & $a, b$ & $b, c$ & $e, c$ \\
$a$ & $a, b$ & $b, c$ & $e, c$ & $e, a$ \\
$b$ & $b, c$ & $e, c$ & $e, a$ & $a, b$ \\
$c$ & $e, c$ & $e, a$ & $a, b$ & $b, c$
\end{tabular}

Let $m$ be a natural number and $\left(Z_{m},+\right)$ be the cyclic group of order $m$. Let $x$ be a real number and $[x]$ be the $m$-class of $x$. Then we obtain the following

Theorem 5.5. [10] Let $n$ be a natural number and set $H_{n}=\{0,1, \cdots, 2 n-1\}$. Then the structure $\left(H_{n}, \oplus\right)$ is a group isomorphic to $\left(Z_{2 n},+\right)$, where $\oplus$ is defined by:

$$
x \oplus y=x+y-2 n[(x+y) / 2 n], \quad \forall(x, y) \in H_{n}^{2} .
$$


Theorem 5.6. [10] Let $n$ be a natural number and $H_{n}=\{0,1, \cdots, 2 n-1\}$. We define an operation $\otimes$ on $H_{n}$ by:

$$
x \otimes y= \begin{cases}x+y-n[(x+y) / n], & \text { if } 0 \leq x<n, \quad 0 \leq y<n \\ x+y-n[(x+y) / n]+n, & \text { if } 0 \leq x<n, \quad n \leq y<2 n \\ x+y-n[(x+y) / n], & \text { if } n \leq x<2 n, n \leq y<2 n \\ x+y-n[(x+y) / n]+n, & \text { if } n \leq x<2 n, \quad 0 \leq y<n\end{cases}
$$

Then $\left(H_{n}, \otimes\right)$ is a group and $\left(H_{n}, \otimes\right) \cong\left(Z_{2} \times Z_{n},+\right)$.

Theorem 5.7. Let $n$ be a natural number and $H_{n}=\{0,1, \cdots, 2 n-1\}$. Then the groups $\left(H_{n}, \oplus\right)$ and $\left(H_{n}, \otimes\right)$ are not mutually associative.

Proof. We prove that there exists $(x, y, z) \in H_{n}^{3}$ such that $(x \oplus y) \otimes z \neq x \otimes(y \oplus z)$. To this end, set $0 \leq x<n, 0 \leq y<n, 0 \leq z<n$, so $0 \leq x+y<2 n$ and $0 \leq y+z<2 n$. If $n \leq x+y<2 n$ and $0 \leq y+z<n$, then $(x \oplus y) \otimes z=(x+y) \otimes z=$ $(x+y)+z-n[(x+y)+z / n]+n$, but $x \otimes(y \oplus z)=x+(y+z)-n[x+(y+z) / n]$. Hence $(x \oplus y) \otimes z \neq x \otimes(y \oplus z)$.

Proposition 5.8. Let $n$ be a natural number and $H_{n}=\{0,1, \cdots, 2 n-1\}$. Then $\left(H_{n}, \star\right)$ is a non-strongly associative hypergroup, where $\star$ is the derived hyperoperation from $\left(H_{n}, \oplus\right)$ and $\left(H_{n}, \otimes\right)$.

Proof. We prove that there exists $(x, y, z) \in H_{n}^{3}$ and $t \in x \star y$ such that for all $s \in y \star z$ we have $t \star z \neq x \star s$. To this end, set $0 \leq x<n, 0 \leq y<n, 0 \leq z<n$, so $0 \leq x+y<2 n$ and $0 \leq y+z<2 n$. If $n \leq x+y<2 n$ and $0 \leq y+z<n$, then $x \star y=\{x+y, x+y-n\}$ and $y \star z=\{y+z\}$. Let $t=x+y-n$. We have $t \star z=\{x+y-n+z\}$, but $x \star(y+z)=\{x+y+z, x+y+z-n\}$. Hence for all $s \in y \star z$, $t \star z \neq x \star s$. Therefore $\left(H_{n}, \star\right)$ is a non-strongly associative hypergroup.

\section{REFERENCES}

[1] Aghabozorgi, H., Jafarpour, M., and Cristea, I., "Complementable semihypergroups", Communications in Algebra, 44 (2016), 1740-1753.

[2] Ameri, R., and Zahedi, M.M., "Hyperalgebraic systems", Italian Journal of Pure and Applied Mathematics, 6 (1999) 21-32.

[3] Corsini, P., Prolegomena of Hypergroup Theory, Second edition, Aviani Editore, 1993.

[4] Corsini, P., and Leoreanu, V., Applications of Hyperstructures Theory, Advanced in Mathematics, Kluwer Academic Publisher, 2003.

[5] Cristea, I., Jafarpour, M., Mousavi, S. Sh., and Soleymani, A.,"Enumeration of Rosenberg hypergroups", Computers and Mathematics with Applications, 60 (2010), 2753-2763, doi:10.1016/j.camwa.2010.09.027.

[6] Davvaz, B., and Leoreanu, V., Hyperring Theory and Applications, Int. Academic Press, 2007.

[7] Davvaz, B., Polygroup theory and related systems, World Sci. Publ., 2013.

[8] Davvaz, B., Semihypergroup Theory, Academic Press, 2016.

[9] Davvaz, B., "A brief survey of the theory of $H_{v}$-structures", 8th Congress Math. Scandenaves, Stockholm, Spanidis Press (2003), 39-70.

[10] Jafarpour, M., Alizadeh, F., "Associated (semi)hypergroups from duplexes", Journal of Algebraic Systems, 2 (2014), 83-96.

[11] Marty, F., Sur une generalization de la notion de group", 8th Congress Math. Scandenaves, Stockholm, (1934), 45-49. 
[12] Rosenberg, I.G.,"Hypergroups and join spaces determined by relations",Italian Journal of Pure and Applied Mathematics, 4 (1998) 93101.

[13] Vougiouklis, T., Hyperstructures and their Representations, Hadronic Press, Inc, 115, Palm Harber, USA, 1994

[14] Vougiouklis, T., The fundamental relation in hyperrings. The general hypereld, Algebraic hyperstructures and applications, (Xanthi, 1990), 203-211, World Sci. Publish- ing, Teaneck, NJ, 1991.

[15] Vougiouklis, T., "Enlarging $H_{v}$-structures", Algebras and Combinatorics, ICAC97, Hong Kong, Springer Verlag, 1999, 455-463.

[16] Vougiouklis, T., "On $H_{v}$-rings and $H_{v}$-representations", Discrete Mathematics, Elsevier, 208/209 (1999), 615-620.

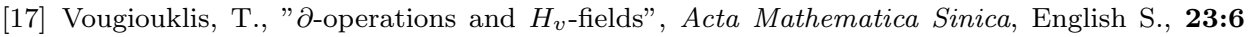
(2008), 965-972. 
F. JANTANI et. al. 